\title{
Fatores associados ao baixo peso em idosos comunitários de sete cidades brasileiras: Estudo FIBRA
}

\author{
Factors associated with being underweight among elderly \\ community-dwellers from seven Brazilian cities: the FIBRA Study
}

Daniela de Assumpção ${ }^{1}$

Flávia Silva Arbex Borim ${ }^{1}$

Priscila Maria Stolses Bergamo Francisco ${ }^{2}$

Anita Liberalesso Neri ${ }^{1}$

${ }^{1}$ Programa de PósGraduação em

Gerontologia, Faculdade de Ciências Médicas, Universidade Estadual de Campinas (Unicamp). R. Tessália Vieira de Camargo 126, Cidade Universitária. 13083-887 Campinas SP Brasil.danideassumpcao@ gmail.com

${ }^{2}$ Departamento de Saúde Coletiva, Faculdade de

Ciências Médicas, Unicamp. Campinas SP Brasil.

\begin{abstract}
The aim of this study was to assess the prevalence of being underweight among the elderly according to demographic and socioeconomic characteristics, health-related behaviors, diseases and health status. This was a cross-sectional multi-center study with 3,478 community-dwelling elders ( $\geq 65$ years). The dependent variable was the prevalence of being underweight, classified by Body Mass Index $<22 \mathrm{~kg} / \mathrm{m} 2$. Adjusted prevalence rate ratios were estimated using multivariable Poisson regression. The mean age was 72.9 years and $12.0 \%$ of the elderly were underweight (CI95\%:10.9-13.1). Aged elderly $\geq 80$ years, former and current smokers, those who reported appetite loss and those classified as pre-frail or frail $(P R=1.41$; CI95\%:1.09-1.82) presented a higher prevalence of being underweight. Individuals who received medical diagnosis of hypertension, diabetes and rheumatism had the lowest underweight prevalence observed. The results highlight the importance of nutritional status assessment and monitoring among the elderly, with emphasis on the most vulnerable subgroups, particularly the frail elderly, taking into account the health consequences of low weight.
\end{abstract}

Key words Elderly, Weight loss, Health of the elderly, Health surveys
Resumo O objetivo foi estimar a prevalência de baixo peso em idosos segundo variáveis demográficas, socioeconômicas, de comportamentos relacionados à saúde, morbidades e estado de saúde. Trata-se de estudo transversal, multicêntrico, que envolveu 3.478 idosos da comunidade ( $\geq 65$ anos). O Índice de Massa Corporal (IMC) foi calculado com informações aferidas de peso e altura. A variável dependente foi o baixo peso, classificada pelo $I M C<22 \mathrm{~kg} / \mathrm{m}^{2}$. Foram estimadas razões de prevalência ajustadas por meio de regressão múltipla de Poisson. A média de idade foi de 72,9 anos e a prevalência de baixo peso atingiu 12,0\% (IC95\%:10,9-13,1) dos indivíduos. Verificaramse prevalencias mais elevadas de baixo peso nos idosos com idade $\geq 80$ anos, nos ex-fumantes $e$ fumantes, naqueles que apresentaram perda de apetite e nos classificados como pré-frágeis ou frágeis $(R P=1,41$; IC95\%:1,09-1,82). Menores prevalências de baixo peso foram observadas nos indivíduos que relataram ter recebido diagnóstico médico de hipertensão, de diabetes e de reumatismo. Os resultados indicam a importância de avaliar e monitorar o estado nutricional dos idosos, com ênfase nos subgrupos mais vulneráveis, em particular nos frágeis, levando em conta as consequências do baixo peso para o estado de saúde. Palavras-chave Idoso, Perda de peso, Saúde do idoso, Inquérito de saúde 


\section{Introdução}

Mudanças fisiológicas e metabólicas ocorrem no organismo durante o processo de envelhecimento com repercussões sobre a saúde e o estado nutricional dos idosos, que se reflete na relação entre peso e altura, e na composição corporal ${ }^{1}$. Tais mudanças tornam o idoso mais suscetível ao desenvolvimento de um quadro de desnutrição e deficiências nutricionais ${ }^{2,3}$. A desnutrição apresenta-se associada à síndrome consumptiva, à sarcopenia, à diminuição de força e à fadiga, ocasionando aumento da mortalidade, da susceptibilidade às infecções e a redução da qualidade de vida dos idosos ${ }^{4}$.

Entre os fatores que favorecem o desenvolvimento do baixo peso nos idosos destacam-se o isolamento social, o menor consumo de alimentos em face de limitações físicas, doenças crônicas, uso de medicamentos que causam inapetência, deterioração cognitiva, depressão, dificuldades de mastigação, alterações do paladar, além dos fatores socioeconômicos e psicológicos que incluem o viver sozinho, a viuvez e a perda da identidade e do papel social ${ }^{5,6}$.

Estudos evidenciam a relação entre baixo peso e mortalidade ${ }^{1,7}$. Dados do Inquérito Saúde, Bem-estar e Envelhecimento (SABE) registraram um risco de óbito 51\% superior nos idosos com baixo peso em relação aos que estavam com o peso adequado ${ }^{8}$.

A literatura tem chamado atenção para a ocorrência de perda da massa magra em associação com a redução da força muscular, fenômeno caracterizado como sarcopenia ${ }^{9}$, condição importante para a definição da síndrome da fragilidade, considerada um desafio para as políticas públicas, por acarretar aumento do risco de ocorrência de quedas, fraturas, incapacidades, dependência, hospitalização e mortalidade ${ }^{10-12}$. A síndrome da fragilidade vem sendo descrita como um estado clínico de aumento da vulnerabilidade, expresso em diminuição das respostas compensatórias e da possibilidade de manutenção da homeostase frente à estressores ${ }^{10}$.

Considerando o cenário de crescimento da população idosa, as mudanças fisiológicas do processo de envelhecimento e as consequências da perda de peso para o estado de saúde, o presente estudo tem como objetivo estimar a prevalência de baixo peso segundo variáveis sociodemográficas, de comportamentos relacionados à saúde, morbidades e estado de saúde em idosos residentes na comunidade.

\section{Métodos}

Trata-se de pesquisa transversal de base populacional que utilizou dados do Estudo de Fragilidade em Idosos Brasileiros (FIBRA), realizado em 2008-2009, em sete cidades brasileiras. Foram coletadas informações de idosos de 65 anos ou mais, recrutados da comunidade e residentes em área urbana.

A amostra do estudo foi obtida por conglomerados e em dois estágios. No primeiro estágio foram sorteados e percorridos os setores censitários da área urbana dos municípios participantes, totalizando 90 em Campinas (São Paulo), 93 em Belém (Pará), 75 em Poços de Caldas (Minas Gerais), 62 em Ermelino Matarazzo (São Paulo), 60 em Campina Grande (Paraíba), 60 em Parnaíba (Piauí) e 27 em Ivoti (Rio Grande do Sul). Em cada área selecionada foram estimadas cotas de homens e mulheres de 65-69, 70-74, 75-79 e 80 anos ou mais, com base na distribuição desses segmentos na população idosa.

No segundo estágio, os idosos foram recrutados nos domicílios por pessoal treinado e devidamente identificado, mediante um roteiro que incluía a apresentação pessoal e da pesquisa, e o convite aos idosos. A equipe de recrutadores identificava os idosos que atendiam os critérios de inclusão, a saber: ter idade igual ou superior a 65 anos, compreender instruções, ser residente permanente no domicílio e aceitar participar do estudo. Foram excluídos os indivíduos que apresentavam déficits de memória, atenção, orientação espacial, temporal e de comunicação, sugestivos de déficit cognitivo; incapacidade permanente ou temporária para andar (exceto os que faziam uso de dispositivo de auxílio à marcha); perda localizada de força e afasia decorrentes de sequela de acidente vascular encefálico; comprometimento grave da motricidade, da fala ou da afetividade associados à doença de Parkinson avançada; déficit auditivo ou visual grave e estar em estágio terminal.

O número mínimo de amostra estimado para cidades com mais de 1 milhão de habitantes, como Campinas e Belém, foi de 601 idosos, considerando um erro de amostragem de 4 pontos percentuais. Para as demais cidades, com menos de 1 milhão de habitantes, a estimativa foi de 384 indivíduos, para um erro amostral de 5\%.

Os dados foram coletados por meio de questionário previamente testado, com questões validadas, aplicado em um único encontro que acontecia em escolas, igrejas, unidades básicas de saúde, clubes e centros de convivência. Estudantes de graduação e de pós-graduação devidamente 
treinados conduziam as entrevistas com duração de 40 a 120 minutos.

Na primeira fase da coleta de dados, da qual participaram todos os idosos selecionados, foram obtidas informações demográficas e socioeconômicas, foram feitas aferições de características antropométricas e de pressão arterial e foi realizada avaliação de fragilidade. O Índice de Massa Corporal (IMC) foi calculado com informações aferidas de peso e altura, por meio da divisão do peso em quilos pelo quadrado da altura em metros. Foi classificado com baixo peso o indivíduo com IMC $<22 \mathrm{~kg} / \mathrm{m}^{2}$, conforme o ponto de corte recomendado para idosos ${ }^{13}$.

Ao final da primeira fase da coleta de dados, foi aplicado um teste de rastreio cognitivo, o Mini Exame do Estado Mental (MEEM). Os pontos de corte adotados para o MEEM foram: 17 para os analfabetos, 22 para os que tinham entre um e quatro anos de escolaridade, 24 para os que possuíam entre cinco e oito anos de estudo e 26 para os escolarizados por nove ou mais anos, menos um desvio-padrão ${ }^{14}$. Os idosos que atingiam pontuações iguais ou superiores às notas de corte do MEEM eram conduzidos à segunda fase da coleta de dados, durante a qual os participantes respondiam questões referentes às doenças crônicas, sinais e sintomas, quedas e fraturas, tabagismo e alcoolismo, autoavaliação da saúde, percepção sobre a saúde bucal e sobre as condições funcionais para a alimentação, sintomas depressivos, satisfação com a vida, acesso a serviços médicos e hospitalares no último ano, entre outras.

No presente estudo foram considerados todos os idosos que tiveram o peso e a altura aferidos, independente da pontuação do MEEM. A variável dependente foi o baixo peso e as variáveis independentes selecionadas para a análise dos fatores associados ao baixo peso foram:

Demográficas e socioeconômicas: sexo, idade, raça/cor da pele autorreferida, situação conjugal, escolaridade (em anos de estudo) e renda familiar per capita (em salários mínimos).

Comportamentos relacionados à saúde e morbidades: tabagismo, frequência de consumo de bebida alcoólica e prática de atividade física em contexto de lazer, avaliada por versão adaptada do Minnesota Leisure Time Physical Activity Questionnarie (MLTPAQ), presença de hipertensão, diabetes, reumatismo, asma, depressão, número de doenças crônicas autorreferidas.

Condições de saúde: perda de apetite, ocorrência de quedas nos últimos doze meses, dificuldades para se alimentar no último ano, comprometimento das funções cognitivas, avaliado pelo MEEM, e fragilidade, considerando-se os cinco critérios propostos por Fried et al. ${ }^{10}$, por meio dos quais se classificaram os idosos como frágeis (positivos para três ou mais critérios), pré-frágeis (positivos para um ou dois critérios) e não-frágeis (para nenhum critério).

Para as análises deste estudo, foram estimadas as prevalências e respectivos intervalos de confiança de $95 \%$. As associações entre variáveis independentes e o baixo peso foram analisadas pelo teste qui-quadrado com nível de significância de 5\%. Também foram produzidas análises de regressão simples e múltipla de Poisson para estimar razões de prevalência brutas e ajustadas. No modelo múltiplo de regressão de Poisson foram inseridas as variáveis que apresentaram nível de significância inferior a 0,20 na análise bivariada, permanecendo no modelo aquelas com $\mathrm{p}<0,05$.

As análises dos dados foram executadas no programa Stata 12.0 (Stata Corp., College Station, Estados Unidos).

O estudo FIBRA foi aprovado pelo Comitê de Ética em Pesquisa com Seres Humanos da Faculdade de Ciências Médicas da Universidade Estadual de Campinas e registrado na Plataforma Brasil.

\section{Resultados}

Foram analisados os dados de 3.478 idosos com idade média de 72,9 anos (IC95\%: 72,7-73,1), sendo de 73,3 anos (IC95\%: 73,0-73,6) para os homens e de 72,8 anos (IC95\%: 72,5-73,0) para as mulheres.

A prevalência de baixo peso na população estudada atingiu $12,0 \%$, sendo significativamente mais elevada nos idosos de 80 anos ou mais, nos sem cônjuge e nos que mencionaram rendimento de até um salário mínimo mensal, comparados aos que recebiam mais que três (Tabela 1 ).

A Tabela 2 mostra maiores prevalências de baixo peso nos idosos que referiram presença de asma, nos pertencentes às categorias de ex-fumante e fumante, e nos que não ingeriam bebida alcoólica ou que ingeriam duas ou mais vezes na semana, comparados aos que utilizavam entre um e quatro vezes no mês. Por outro lado, menores prevalências de baixo peso foram verificadas nos indivíduos que relataram ter recebido diagnóstico médico de hipertensão, diabetes, reumatismo, e nos que apresentavam duas ou mais doenças crônicas.

Os idosos que apresentaram perda de apetite e pré-fragilidade ou fragilidade tiveram prevalências mais altas de baixo peso (Tabela 3 ).

A Tabela 4 demonstra os resultados da análise múltipla de regressão de Poisson. Verificou-se 
Tabela 1. Prevalência e razão de prevalência de baixo peso em idosos de 65 anos ou mais, segundo variáveis sociodemográficas. Estudo FIBRA UNICAMP (2008/2009).

\begin{tabular}{|c|c|c|c|c|}
\hline Variáveis & n (\%) & Prevalência & RP bruta (IC95\%) & RP ajustada ${ }^{* *}(\mathrm{IC} 95 \%)$ \\
\hline Sexo & & $0,061^{*}$ & & \\
\hline Masculino & $1.125(32,35)$ & 13,51 & 1 & 1 \\
\hline Feminino & $2.353(67,65)$ & 11,30 & $0,83(0,68-1,02)$ & $0,85(0,69-1,03)$ \\
\hline Total & 3.478 & 12,02 & & \\
\hline Faixa etária (em anos) & & $<0,001^{*}$ & & \\
\hline 65 a 69 & $1.206(34,68)$ & 10,70 & 1 & 1 \\
\hline 70 a 74 & $1.056(30,37)$ & 10,42 & $0,97(0,75-1,25)$ & $0,96(0,74-1,24)$ \\
\hline 75 a 79 & $687(19,75)$ & 12,08 & $1,13(0,86-1,48)$ & $1,12(0,85-1,48)$ \\
\hline $80 \mathrm{e}+$ & $528(15,18)$ & 18,18 & $1,70(1,30-2,21)$ & $1,67(1,28-2,18)$ \\
\hline Raça / Cor da pele & & $0,096^{*}$ & & \\
\hline Branca & $1.800(52,26)$ & 11,11 & 1 & 1 \\
\hline Não branca & $1.644(47,73)$ & 12,96 & $1,16(0,96-1,41)$ & $1,17(0,97-1,42)$ \\
\hline Estado conjugal & & $0,035^{*}$ & & \\
\hline Com cônjuge & $1.666(48,00)$ & 10,80 & 1 & 1 \\
\hline Sem cônjuge & $1.805(52,00)$ & 13,13 & $1,21(1,00-1,47)$ & $1,28(1,03-1,59)$ \\
\hline Escolaridade (em anos) & & $0,512^{*}$ & & \\
\hline 0 & $699(20,12)$ & 13,30 & $1,14(0,87-1,49)$ & $1,07(0,82-1,40)$ \\
\hline 1 a 4 & $1.709(49,22)$ & 11,76 & $1,01(0,80-1,26)$ & $1,00(0,80-1,25)$ \\
\hline $5 \mathrm{ou}+$ & $1.065(30,66)$ & 11,64 & 1 & 1 \\
\hline $\begin{array}{l}\text { Renda per capita } \\
\text { (em salários mínimos) }\end{array}$ & & $<0,001^{*}$ & & \\
\hline$\leq 1$ & $380(12,81)$ & 17,11 & $1,82(1,34-2,49)$ & $1,82(1,33-2,48)$ \\
\hline$>1 \mathrm{a} \leq 3$ & $1.467(49,41)$ & 11,86 & $1,26(0,99-1,61)$ & $1,27(1,00-1,62)$ \\
\hline$>3$ & $1.122(37,78)$ & 9,36 & 1 & 1 \\
\hline
\end{tabular}

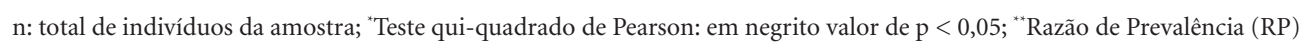
ajustada por sexo e/ou idade.

maior razão de prevalência com o baixo peso nas categorias de 80 anos ou mais ( $R P=1,61)$, ex-fumante $(\mathrm{RP}=2,06)$ e fumante $(\mathrm{RP}=1,32)$, perda de apetite $(\mathrm{RP}=1,83)$ e algum grau de fragilidade $(\mathrm{RP}=1,41)$ quando comparados às respectivas categorias de referência. Os segmentos de idosos hipertensos, diabéticos e portadores de doenças reumáticas apresentaram $43 \%, 54 \%$ e $42 \%$, respectivamente, menores prevalências de baixo peso em relação às categorias de referência.

\section{Discussão}

Neste estudo, a prevalência de baixo peso teve valor um pouco inferior ao observado em Viçosa/MG (13,6\%) em amostra de 621 idosos de 60 anos ou mais ${ }^{15}$; e em Bambuí/MG (14,4\%) em pesquisa que analisou 1.443 indivíduos $(\geq 60$ anos) utilizando o IMC $\leq 20 \mathrm{~kg} / \mathrm{m}^{2}$ como critério para a definição de baixo peso ${ }^{16}$. Entre idosos longevos ( 80 anos ou mais), Boscatto et al. ${ }^{17}$ encontraram prevalência de 18,8\% em Antônio Carlos/
SC. Em idosos institucionalizados, as estimativas são ainda mais elevadas, alcançando $58,8 \%$, em Uberlândia/ $\mathrm{MG}^{4}$, e 53,1\%, em Fortaleza/CE ${ }^{18}$.

Não foi observada diferença estatisticamente significativa entre baixo peso e sexo, contradizendo os achados de outras pesquisas que apontam maior ocorrência no sexo masculino ${ }^{15,16}$.

A prevalência de baixo peso foi mais alta nos idosos de 80 anos ou mais $(18,2 \%)$, do que entre os de 65 a 69 anos (10,7\%). Os grupos de idosos mais velhos tendem a apresentar maiores taxas de baixo peso do que os de idosos jovens, conforme verificado em outros estudos ${ }^{16,17,19}$. Segundo Villareal et al. ${ }^{20}$, a constatação de que o peso tende a diminuir após os 60 anos pode decorrer de viés de sobrevivência, já que resultados de estudos longitudinais não indicam alteração significativa no peso.

Comparados aos que nunca fumaram, os fumantes $(\mathrm{RP}=1,32)$ e os ex-fumantes $(\mathrm{RP}=2,06)$ apresentaram maiores proporções de baixo peso, replicando os dados de Barreto et al. ${ }^{16}$. No Inquérito de Saúde no Estado de São Paulo (ISA-SP), Zaitune et al. ${ }^{21}$ observaram prevalência elevada 
Tabela 2. Prevalência e razão de prevalência de baixo peso em idosos de 65 anos ou mais, segundo variáveis de comportamentos relacionados à saúde e morbidades. Estudo FIBRA UNICAMP (2008/2009).

\begin{tabular}{|c|c|c|c|c|}
\hline Variáveis & n (\%) & Prevalência & RP bruta (IC95\%) & RP ajustada ${ }^{* *}($ IC95\%) \\
\hline Hipertensão & & $<0,001^{*}$ & & \\
\hline Não & $991(37,94)$ & 16,75 & 1 & 1 \\
\hline Sim & $1.621(62,06)$ & 8,02 & $0,47(0,38-0,60)$ & $0,49(0,39-0,61)$ \\
\hline Diabetes & & $<0,001^{*}$ & & \\
\hline Não & $2.068(79,17)$ & 12,96 & 1 & 1 \\
\hline Sim & $544(20,83)$ & 5,33 & $0,41(0,28-0,60)$ & $0,41(0,28-0,61)$ \\
\hline Reumatismo & & $<0,001^{*}$ & & \\
\hline Não & $1.469(56,40)$ & 13,89 & 1 & 1 \\
\hline Sim & $1.136(43,60)$ & 8,10 & $0,58(0,45-0,74)$ & $0,59(0,46-0,76)$ \\
\hline Asma & & $0,001^{*}$ & & \\
\hline Não & $2.334(89,53)$ & 10,67 & 1 & 1 \\
\hline Sim & $273(10,47)$ & 17,22 & $1,61(1,18-2,20)$ & $1,60(1,17-2,18)$ \\
\hline Número de morbidades & & $<0,001^{*}$ & & \\
\hline 0 a 1 & $981(38,05)$ & 15,70 & 1 & 1 \\
\hline $2 \mathrm{ou}+$ & $1.597(61,95)$ & 8,64 & $0,55(0,43-0,69)$ & $0,55(0,44-0,70)$ \\
\hline Depressão & & $0,076^{*}$ & & \\
\hline Sim & $539(20,68)$ & 9,09 & $0,77(0,56-1,04)$ & $0,80(0,59-1,09)$ \\
\hline Não & $2.068(79,32)$ & 11,80 & 1 & 1 \\
\hline Tabagismo & & $<0,001^{*}$ & & \\
\hline Nunca fumou & $1.386(53,12)$ & 8,80 & 1 & 1 \\
\hline Ex-fumante & $979(37,53)$ & 12,26 & $1,39(1,08-1,79)$ & $1,36(1,04-1,78)$ \\
\hline Fumante & $244(9,35)$ & 22,54 & $2,56(1,86-3,52)$ & $2,64(1,90-3,66)$ \\
\hline Frequência de consumo de álcool & & $0,005^{*}$ & & \\
\hline Não bebe & $1.813(70,46)$ & 12,13 & $1,52(1,11-2,08)$ & $1,55(1,13-2,14)$ \\
\hline Bebe de 1 a 4 vezes no mês & $590(22,93)$ & 7,97 & 1 & 1 \\
\hline Bebe 2 ou + vezes na semana & $170(6,61)$ & 15,29 & $1,92(1,19-3,09)$ & $1,75(1,08-2,83)$ \\
\hline Prática de atividade física & & $0,399^{*}$ & & \\
\hline Ativo & $1.128(32,44)$ & 11,35 & 1 & 1 \\
\hline Não ativo & $2.350(67,56)$ & 12,34 & $1,09(0,88-1,33)$ & $1,06(0,86-1,31)$ \\
\hline
\end{tabular}

n: total de indivíduos da amostra; ${ }^{*}$ Teste qui-quadrado de Pearson: em negrito valor de $\mathrm{p}<0,05$; ${ }^{* *}$ Razão de prevalência (RP) justada por sexo e idade.

de fumantes (RP = 1,91; IC95\%: 1,42-2,57) entre os idosos com baixo peso. Pesquisa de coorte realizada com 70.394 chineses adultos e idosos, verificou nos tabagistas probabilidade 2,7\% maior de apresentarem baixo peso comparados aos não tabagistas $^{22}$. A relação entre tabagismo e baixo peso tem sido atribuída à nicotina, por desencadear aumento da atividade adrenérgica e dos níveis cerebrais de neurotransmissores anorexígenos, como a serotonina e dopamina, que atuam na regulação do apetite ${ }^{23,24}$.

O baixo peso mostrou-se superior nos indivíduos que relataram redução do apetite. Diversos fatores interferem na ingestão alimentar e no estado nutricional dos idosos, incluindo a anorexia fisiológica do envelhecimento, provocada pela elevação plasmática do hormônio colecistoqui- nina, que regula a saciedade ${ }^{25}$; alterações sensoriais, perda de dentes, isolamento social, depressão e baixo nível socioeconômico são condições que afetam a autonomia dos indivíduos para selecionar e preparar os alimentos, limitando o repertório alimentar e o interesse pela comida ${ }^{6,26}$. A falta de apetite associada à monotonia alimentar pode gerar deficiências de nutrientes essenciais à saúde dos idosos, tais como o zinco que, juntamente com outras vitaminas e compostos fitoquímicos, atua na cicatrização de feridas, na prevenção da degeneração macular e na melhora da percepção de sabores ${ }^{3,26}$. Dados do Inquérito Nacional de Alimentação, conduzido em 20082009 com 4.322 idosos ( $\geq 60$ anos), revelaram elevadas prevalências de inadequação de cálcio, magnésio e vitaminas A, C, D e $\mathrm{E}^{27}$. 
Tabela 3. Prevalência e razão de prevalência de baixo peso em idosos de 65 anos ou mais, segundo variáveis de condições de saúde. Estudo FIBRA UNICAMP (2008/2009).

\begin{tabular}{lcccc}
\hline \multicolumn{1}{c}{ Variáveis } & $\mathbf{n}(\mathbf{\%})$ & Prevalência $^{*}$ & RP bruta (IC95\%) & RP ajustada* $^{* *}$ (IC95\%) \\
\hline Perda de apetite & & $<\mathbf{0 , 0 0 1}^{*}$ & & \\
Sim & $650(25,11)$ & 17,69 & $1,92(1,52-2,43)$ & $1,95(1,54-2,48)$ \\
Não & $1.938(74,89)$ & 9,18 & 1 & 1 \\
Queda & & $0,390^{*}$ & & \\
Sim & $736(29,45)$ & 10,60 & $0,90(0,69-1,16)$ & $0,91(0,70-1,18)$ \\
Não & $1.763(70,55)$ & 11,80 & 1 & 1 \\
Fragilidade & & $<\mathbf{0 , 0 0 1 ^ { * }}$ & & 1 \\
Não & $1.334(39,06)$ & 8,85 & 1 & $1,46(1,17-1,83)$ \\
Pré-frágil & $1.770(51,83)$ & 13,45 & $1,52(1,22-1,89)$ & $1,57(1,11-2,22)$ \\
Frágil & $311(9,11)$ & 15,43 & $1,74(1,25-2,44)$ & 1 \\
Dificuldade para se alimentar & & $0,095^{*}$ & & $1,24(0,94-1,64)$ \\
Não & $748(33,42)$ & 9,22 & 1 & $1,14(0,91-1,41)$ \\
Sim & $1.490(66,58)$ & 11,54 & $1,25(0,94-1,65)$ & 1 \\
MEEM & & $0,058^{*}$ & & $1,21(0,98-1,49)$ \\
$\quad$ Com déficit & $882(25,40)$ & 13,83 & 1 & 1,42 \\
Sem déficit & $2.591(74,60)$ & $11,42)$ & \\
\hline
\end{tabular}

n: total de indivíduos da amostra; ${ }^{*}$ Teste qui-quadrado de Pearson: em negrito valor de $\mathrm{p}<0,05$; ${ }^{* *}$ Razão de prevalência (RP) ajustada por sexo e idade.

Tabela 4. Modelo múltiplo com regressão de Poisson: variáveis associadas ao baixo peso em idosos de 65 anos ou mais. Estudo FIBRA UNICAMP (2008/2009).

\begin{tabular}{|c|c|c|c|}
\hline Variáveis & $\mathbf{R P}^{*}$ & IC95\% & Valor de $\mathbf{p}^{* *}$ \\
\hline \multicolumn{4}{|l|}{ Faixa etária (em anos) } \\
\hline 65 a 69 & 1 & & \\
\hline 70 a 74 & 1,10 & $0,82-1,48$ & 0,523 \\
\hline 75 a 79 & 1,13 & $0,81-1,59$ & 0,444 \\
\hline $80 \mathrm{e}+$ & 1,61 & $1,14-2,26$ & 0,006 \\
\hline \multicolumn{4}{|l|}{ Hipertensão } \\
\hline Não & 1 & & \\
\hline Sim & 0,57 & $0,45-0,73$ & $<0,001^{*}$ \\
\hline \multicolumn{4}{|l|}{ Diabetes } \\
\hline Não & 1 & & \\
\hline Sim & 0,46 & $0,31-0,68$ & $<0,001^{\star}$ \\
\hline \multicolumn{4}{|l|}{ Reumatismo } \\
\hline Não & 1 & & \\
\hline Sim & 0,58 & $0,45-0,76$ & $<0,001^{*}$ \\
\hline \multicolumn{4}{|l|}{ Perda de apetite } \\
\hline Não & 1 & & \\
\hline Sim & 1,83 & $1,43-2,34$ & $<0,001^{*}$ \\
\hline \multicolumn{4}{|l|}{ Tabagismo } \\
\hline Nunca fumou & 1 & & \\
\hline Ex-fumante & 2,06 & $1,47-2,89$ & $<0,001^{*}$ \\
\hline Fumante & 1,32 & $1,00-1,73$ & 0,045 \\
\hline \multicolumn{4}{|l|}{ Fragilidade } \\
\hline Não & 1 & & \\
\hline Pré-frágil/frágil & 1,41 & $1,09-1,82$ & 0,008 \\
\hline
\end{tabular}

"Razão de prevalência (RP) ajustada por todas as variáveis da tabela e por sexo; * Em negrito valor de p < 0,05. 
Nesta pesquisa, os idosos classificados como frágeis ou pré-frágeis apresentaram maior ocorrência de baixo peso do que os não-frágeis. Analisando 3.075 participantes do estudo FIBRA, Moretto et al..$^{28}$ observaram um valor médio de IMC significativamente menor nos idosos frágeis do que nos não-frágeis. A fragilidade é uma síndrome clínica associada ao envelhecimento fisiológico normal e agravada pelos danos acumulados ao longo da vida ${ }^{29}$. Neste estudo, se destaca como variável antecedente à perda de peso e ao baixo status nutricional.

Menores prevalências de baixo peso foram identificadas nos idosos que referiram hipertensão, diabetes e doenças reumáticas. Dados da coorte de idosos de Bambuí/MG revelam menores prevalências de baixo peso entre os idosos diagnosticados com hipertensão e diabetes ${ }^{16} . \mathrm{O}$ excesso de peso e a obesidade, em todas as faixas etárias, levam ao aumento da morbimortalidade por doenças crônicas, entre elas a hipertensão arterial sistêmica e outras doenças cardiovasculares, diabetes mellitus, certos tipos de neoplasia, síndrome de apneia/hipopneia do sono e osteoartrite ${ }^{30,31}$. Sabe-se que o envelhecimento determina diversas modificações na composição corporal e se associa ao aumento da massa gordurosa e a mudanças no seu padrão de distribuição ${ }^{1}$. Um quadro preocupante é o desenvolvimento da obesidade sarcopênica, caracterizada pela coexistência de excesso de gordura e de redução da massa magra ${ }^{32}$.

Recomendar a perda de peso em idosos não é tarefa simples, pois deve ser mantida uma alimentação de elevada densidade nutricional e adequada densidade energética, associada à prática de atividade física regular ${ }^{26}$. As modificações no estilo de vida, principalmente nesta fase da vida, exigem grande motivação dos idosos e dos profissionais de saúde. É necessário que os serviços de saúde tenham capacidade de apoiar esse processo de mudança com orientações práticas sobre como enfrentar as dificuldades encontradas no cotidiano. A educação em saúde, prática que visa a transformação dos modos de vida dos indivíduos, pode ser aplicada nas estratégias de promoção do envelhecimento saudável trazendo resultados relevantes à saúde dos idosos ${ }^{33}$.

Os idosos apresentam diversas alterações fisiológicas naturais do processo de envelhecimento, que os tornam suscetíveis ao desenvolvimento de distúrbios nutricionais que podem afetar o seu estado de saúde ${ }^{34}$. O contexto de vida em que se insere a maioria dos idosos brasileiros, sujeitos aos efeitos cumulativos acarretados pelo baixo nível econômico, baixa escolaridade e ausência de suporte social, exige a incorporação de uma abordagem de cuidado ampliado. A alimentação tem papel importante neste processo, principalmente, na etiologia de doenças associadas à idade, ao declínio funcional e ao surgimento de deficiências ${ }^{35}$.

Avaliações do estado nutricional, do comportamento alimentar e das condições sociais em que os idosos estão inseridos, considerando a complexa rede de determinantes diretos e indiretos do envelhecimento e da saúde na velhice podem subsidiar ações favoráveis à melhoria da qualidade de vida e à promoção do envelhecimento saudável.

\section{Colaboradores}

D Assumpção realizou a proposta do artigo, revisão da literatura, análise dos dados e redação do texto. FSA Borim e PMSB Francisco participaram da análise, interpretação dos dados e redação do artigo. AL Neri realizou a revisão crítica do manuscrito e aprovou sua versão final para publicação.

\section{Agradecimentos}

Ao Conselho Nacional de Desenvolvimento Científico e Tecnológico pelo financiamento da pesquisa. À CAPES pela bolsa de pós-doutorado concedida a D Assumpção e FSA Borim.

\section{Referências}

1. Gaddey HL, Holder K. Unintentional weight loss in older adults. Am Fam Physician 2014; 89(9):718-722.

2. Amarya S, Singh K, Sabharwal M. Changes during aging and their association with malnutrition. J Clin Gerontol Geriatr 2015; 6(3):78-84.

3. Brownie $S$. Why are elderly individuals at risk of nutritional deficiency? Int J Nurs Pract 2006; 12(2):110-118.

4. Sousa KT, Mesquita LAS, Pereira LA, Azeredo CM. Baixo peso e dependência funcional em idosos institucionalizados de Uberlândia (MG), Brasil. Cien Saude Colet 2014; 19(8):3513-3520.

5. Ferreira LS, Amaral TF, Marucci MFN, Nascimento LFC, Lebrão ML, Duarte YAO. Undernutrition as a major risk factor for death among older Brazilian adults in the community-dwelling setting: SABE survey. Nutrition 2011; 10:1017-1022. 
6. Stajkovic S, Aitken EM, Holroyd-Leduc J. Unintentional weight loss in older adults. CMAJ 2011; 183(4):443449.

7. Morley JE. Undernutrition in older adults. Family Practice 2012; 29(Supl. 1):i89-i93.

8. Suemoto CK, Lebrão ML, Duarte YA, Danaei G. Effects of body mass index, abdominal obesity, and type 2 diabetes on mortality in community-dwelling elderly in São Paulo, Brazil: analysis of prospective data from the SABE Study. J Gerontol A Biol Sci Med Sci 2015; 70(4):503-510.

9. Bianchi L, Ferrucci L, Cherubini A, Maggio M, Bandinelli S, Savino E, Brombo G, Zuliani G, Guralnik JM, Landi F, Volpato S. The Predictive Value of the EWGSOP Definition of Sarcopenia: Results from the InCHIANTI Study. J Gerontol A Biol Sci Med Sci 2016; 71(2):259-264.

10. Fried LP, Tangen CM, Walston J, Newman AB, Hirsch C, Gottdiener J, Seeman T, Tracy R, Kop WJ, Burke G, McBurnie MA; Cardiovascular Health Study Collaborative Research Group. Frailty in older adults: evidence for a phenotype. J Gerontol A Biol Sci Med Sci 2001; 56(3):M146-156.

11. Lang T, Streeper T, Cawthon P, Baldwin K, Taaffe DR, Harris TB. Sarcopenia: etiology, clinical consequences, intervention, and assessment. Osteoporos Int 2010; 21(4):543-559.

12. Walston JD. Sarcopenia in older adults. Curr Opin Rheumatol 2012; 24(6):623-627.

13. Cervi A, Franceschini SCC, Priore SE. Análise crítica do uso do índice de massa corporal para idosos. Rev Nutr 2005; 18(6):765-775.

14. SMD, Nitrini R, Caramelli P, Bertolucci PH, Okamoto IH. Sugestões para o uso do Mini-Exame do Estado Mental no Brasil. Arq Neuropsiquiatr 2003; 61(3B):777781.

15. Nascimento CM, Ribeiro AQ, Cotta RMM, Acurcio FA, Peixoto SV, Priore SE, Franceschini SCC. Estado nutricional e fatores associados em idosos do Município de Viçosa, Minas Gerais, Brasil. Cad Saude Publica 2011; 27(12):2409-2418.

16. Barreto SM, Passos VMA, Lima-Costa MFF. Obesity and underweight among Brazilian elderly. The Bambuí Health and Aging Study. Cad Saude Publica 2003; 19(2):605-612.

17. Boscatto EC, Duarte MFS, Coqueiro RS, Barbosa AR. Nutritional status in the oldest elderly and associated factors. Rev Assoc Med Bras 2013; 59(1):40-47.

18. Menezes TN, Marucci MFN. Avaliação antropométrica de idosos residentes em Instituições deLonga Permanência de Fortaleza-CE. Rev Bras Geriatr Gerontol 2010; 13(2):235-243.

19. St-Onge MP, Gallagher D. Body composition changes with aging: The cause or the result of alterations in metabolic rate and macronutrient oxidation? Nutrition 2010; 26(2):152-155.

20. Villareal DT, Apovian CM, Kushner RF, Klein S. Obesity in older adults: technical review and position statement of the American Society for Nutrition and NAASO, The Obesity Society. Am J Clin Nutr 2005; 82(5):923-934.

21. Zaitune MPA, Barros MBA, Lima MG, César CLG, Carandina L, Goldbaum M, Alves MCGP. Fatores associados ao tabagismo em idosos: Inquérito de Saúde no Estado de São Paulo (ISA-SP). Cad Saude Publica 2012; 28(3):583-595.
22. Wang Q. Smoking and body weight: evidence from China health and nutrition survey. BMC Public Health 2015; 15:1238.

23. Koopmann A, Bez J, Lemenager T, Hermann D, Dinter C, Reinhard I, Hoffmann H, Wiedemann K, Winterer G, Kiefer F. Effects of Cigarette Smoking on Plasma Concentration of the Appetite-Regulating Peptide Ghrelin. Ann Nutr Metab 2015; 66(2-3):155-161.

24. Klein LC, Corwin EJ, Ceballos RM. Leptin, hunger, and body weight: influence of gender, tobacco smoking, and smoking abstinence. Addict Behav 2004; 29(5):921-927.

25. Landi F, Calvani R, Tosato M, Martone AM, Ortolani E, Savera G, Sisto A, Marzetti E. Anorexia of Aging: Risk Factors, Consequences, and Potential Treatments. Nutrients 2016; 8(2):1-10.

26. Clifford J, Bellows L. Nutrition and Aging. Colorado State University. Fact Sheet no 9.322. Disponível em: http://extension.colostate.edu/docs/pubs/foodnut 109322.pdf.

27. Fisberg RM, Marchioni DML, Castro MA, Verly Junior E, Araujo MC, Bezerra IN, Pereira RA, Sichieri R. Ingestão inadequada de nutrientes na população de idosos do Brasil: Inquérito Nacional de Alimentação 20082009. Rev Saude Publica 2013; 47(Supl. 1):222-230.

28. Moretto MC, Alves RMA, Neri AL, Guariento ME. Relação entre estado nutricional e fragilidade em idosos brasileiros. Rev Bras Clin Med 2012; 10(4):267-271.

29. Neri AL. Palavras-chave em Gerontologia. Brasília: Ed. Alínea; 2014.

30. World Health Organization (WHO). Global status report on noncommunicable diseases 2010. Geneva: WHO; 2011.

31. World Health Organization (WHO). The challenge of obesity in the WHO European Region and the strategies for response. Copenhagen: WHO; 2007.

32. Li Z, Heber D. Sarcopenic obesity in the elderly and strategies for weight management. Nutr Rev 2012; 70(1):57-64.

33. Mallmann DG, Galindo Neto NM, Sousa JC, Vasconcelos EMR. Educação em saúde como principal alternativa para promover a saúde do idoso. Cien Saude Colet 2015; 20(6):1763-1772.

34. Cortez ACL, Carvalho e Martins MC. Indicadores antropométricos do estado nutricional em idosos: revisão sistemática. Ciênc Ciênc Biol Saúde 2012; 14(4):271277.

35. Inzitari M, Doets E, Bartali B, Benetou V, DiBari M, Visser M, Volpato S, Gambassi G, Topinkova E, De Groot L, Salva A; International Association Of Gerontology And Geriatrics (IAGG) Task Force For Nutrition In The Elderly. Nutrition in the age-related disablement process. J Nutr Health Aging 2011; 15(8):599-604.

Artigo apresentado em 05/04/2016

Aprovado em 04/07/2016

Versão final apresentada em 06/07/2016 